CERN-TH/95-322

ENSLAPP-A-566/95

THU-95/34

hep-th/9601087

\title{
TODA FIELDS OF SO(3) HYPER-KAHLER METRICS
}

\author{
Ioannis Bakas 周 \\ Theory Division, CERN, 1211 Geneva 23, Switzerland, and \\ Laboratoire de Physique Theorique ENSLAPP, 74941 Annecy-le-Vieux, France \\ Konstadinos Sfetsos 3 \\ Institute for Theoretical Physics, Utrecht University \\ Princetonplein 5, TA 3508, The Netherlands
}

\begin{abstract}
We examine the Toda frame formulation of the $S O(3)$-invariant hyper-Kahler 4-metrics, namely Eguchi-Hanson, Taub-NUT and Atiyah-Hitchin. Our method exploits the presence of a rotational $S O(2)$ isometry, leading to the explicit construction of all three complex structures as a singlet plus a doublet. The Atiyah-Hitchin metric on the moduli space of BPS $S U(2)$ monopoles with magnetic charge 2 is purely rotational.

Contribution to the proceedings of the 29th International Symposium Ahrenshoop on the Theory of Elementary Particles, Buckow, Germany, 29 August - 2 September 1995 (invited talk given by I. Bakas); to appear in Nuclear Physics B Supplement
\end{abstract}

December 1995

\footnotetext{
*Permanent address: Department of Physics, University of Patras, 26110 Patras, Greece

${ }^{\dagger} \mathrm{e}-\mathrm{mail}$ address: BAKAS@SURYA11.CERN.CH, BAKAS@LAPPHP8.IN2P3.FR

${ }^{\ddagger}$ Present address

$\S$ e-mail address: SFETSOS@FYS.RUU.NL
} 
Hyper-Kahler geometry is a much studied subject in modern theoretical physics, especially in connection with the theory of gravitational instantons, supersymmetric models and supergravity, and various moduli problems in monopole physics, string theory and elsewhere. The hyper-Kahler condition is equivalent to the self-duality (or anti-selfduality) of a metric in four dimensions, which is in turn equivalent to Ricci flatness plus the Kahler condition. Hyper-Kahler spaces admit three independent complex structures $I, J, K$, satisfying the quaternion algebra identities,

$$
I^{2}=J^{2}=K^{2}=-1, \quad I J=-J I=K, \quad \text { etc. }
$$

and hence there is a whole sphere of complex structures generated by $\alpha I+\beta J+\gamma K$, provided that $\alpha^{2}+\beta^{2}+\gamma^{2}=1$. Because of this property, twistor theory provides a natural framework for studying the algebraic properties and the explicit construction of hyper-Kahler metrics [1]. In this paper we study metrics with isometries that do not preserve the complex structures, in that the action of the corrresponding Killing vector fields on the complex structures is non-tri-holomorphic; such isometries are also known in the literature as rotational. Our main interest in this subject arises from string theory, where T-duality transformations with respect to rotational isometries lead to non-local realizations of the $N=4$ world-sheet supersymmetry using parafermion-like variables in the dual formulation of certain superstring vacua [2]. Instead of adopting the minitwistor approach for hyper-Kahler metrics with isometries, we will use in our discussion a local description in terms of adapted coordinates, where the rotational isometry is manifest, and the self-duality condition on the metric becomes a non-linear differential equation in the three (reduced) dimensions, known as the continual Toda equation.

We focus on the special class of 4-dim hyper-Kahler metrics with $S O(3)$ symmetry, whose line element in the Bianchi IX formalism is

$$
d s^{2}=f^{2}(t) d t^{2}+a^{2}(t) \sigma_{1}^{2}+b^{2}(t) \sigma_{2}^{2}+c^{2}(t) \sigma_{3}^{2} .
$$

Here, $\sigma_{1}, \sigma_{2}, \sigma_{3}$ are the invariant 1 -forms of $S O(3)$,

$$
\begin{aligned}
\sigma_{1} & =\frac{1}{2}(\sin \psi d \theta-\sin \theta \cos \psi d \phi), \\
\sigma_{2} & =-\frac{1}{2}(\cos \psi d \theta+\sin \theta \sin \psi d \phi), \\
\sigma_{3} & =\frac{1}{2}(d \psi+\cos \theta d \phi),
\end{aligned}
$$

where $\theta, \psi, \phi$ are the Euler angles, and the normalization has been chosen so that $\sigma_{i} \wedge \sigma_{j}=\frac{1}{2} \epsilon_{i j k} d \sigma_{k}$. The coordinate $t$ of the metric can always be chosen so that

$$
f(t)=\frac{1}{2} a b c,
$$

using a suitable reparametrization. We already see that $\partial / \partial \phi$ is a manifest Killing vector field. The Killing coordinates associated with the other two generators of $S O(3)$ can 
not be made simultaneously manifest, since otherwise they would commute with $\partial / \partial \phi$, contrary to the non-abelian nature of the $S O(3)$ isometry group. It may also happen that specific solutions of the form (2) exhibit additional isometries, which is indeed the case as we will see in the following.

It was established some time ago [3] that the second-order differential equations that provide the self-duality condition for the class of metrics (2), can be integrated once to yield the following first-order system in $t$ :

$$
\begin{aligned}
2 \frac{a^{\prime}}{a} & =b^{2}+c^{2}-2 \lambda_{1} b c-a^{2}, \\
2 \frac{b^{\prime}}{b} & =c^{2}+a^{2}-2 \lambda_{2} c a-b^{2}, \\
2 \frac{c^{\prime}}{c} & =a^{2}+b^{2}-2 \lambda_{3} a b-c^{2},
\end{aligned}
$$

where the three parameters $\lambda_{i}$ remain undetermined for the moment. The derivatives (denoted by prime) are taken with respect to $t$, which satisfies the constraint (4), and there is also an overall sign ambiguity in (5) depending on the self-dual or the anti-selfdual character of the metric; the two cases are related to each other by leting $t \rightarrow-t$. Next we present the only three solutions that exist in this class leading to complete, regular, $S O(3)$-invariant hyper-Kahler 4-metrics, namely Eguchi-Hanson, Taub-NUT and Atiyah-Hitchin.

(i) Eguchi-Hanson metric : This metric corresponds to $\lambda_{1}=\lambda_{2}=\lambda_{3}=0$, and its standard form is given by the line element

$$
d s_{E H}^{2}=\frac{d r^{2}}{1-\left(\frac{m}{r}\right)^{4}}+r^{2}\left(\sigma_{1}^{2}+\sigma_{2}^{2}+\left(1-\left(\frac{m}{r}\right)^{4}\right) \sigma_{3}^{2}\right)
$$

where $m$ is the moduli parameter (for a review, see for instance [4]). For $m=0$ we obtain the flat space limit of the metric in a manifest $S O(3)$-invariant notation. The coordinate $r$ is related to $t$, satisfying the normalization (4), by

$$
r^{2}=m^{2} \operatorname{coth}\left(m^{2} t\right)
$$

(ii) Taub-NUT metric : This metric corresponds to $\lambda_{1}=\lambda_{2}=\lambda_{3}=1$, and its standard form is given by the line element

$$
d s_{T N}^{2}=\frac{1}{4} \frac{r+m}{r-m} d r^{2}+\left(r^{2}-m^{2}\right)\left(\sigma_{1}^{2}+\sigma_{2}^{2}\right)+4 m^{2} \frac{r-m}{r+m} \sigma_{3}^{2},
$$

where $m$ is the relevant moduli parameter (for a review, see for instance [4]). Again the coordinate $r$ does not satisfy the normalization (4), but it is related to $t$ by

$$
r=m+\frac{1}{2 m t}
$$


Note that these two solutions exhibit a bigger group of isometries $S O(3) \times S O(2)$ because $a^{2}=b^{2}$, and hence $\partial / \partial \psi$ generates an additional manifest isometry that commutes with the generators of $S O(3)$. It was thought until 1985 that these two metrics exhaust the list of complete $S O(3)$-invariant hyper-Kahler 4-metrics [3]. However, it also became clear at that time that the moduli space $M_{2}^{0}$ of BPS $S U(2)$ monopoles of magnetic charge 2 defines a new hyper-Kahler metric with $S O(3)$ isometry. Geodesics in $M_{2}^{0}$ describe the motion of slowly moving monopoles, and certain physical arguments due to Manton claimed that the scattering of such monopoles can generate electric charge, thus converting monopoles into dyons [5]. On the other hand, it was known that the metric on the moduli space of well-separated monopoles can be approximated by the Taub-NUT limit, which exhibits an additional $S O(2)$ isometry coming from $a^{2}=b^{2}$. To prove Manton's conjecture as the two monopoles come close to each other, one had to find a new solution with all metric coefficients $a, b, c$ unequal, so that the absence of such an additional $S O(2)$ isometry could explain the generation of electric charge. The metric in question was finally found in closed form by Atiyah and Hitchin [6] (but see also [7]), and it was further shown that together with the Eguchi-Hanson and Taub-NUT metrics, these three solutions complete the classification of the regular hyper-Kahler 4-metrics with $S O(3)$ symmetry [8]. Other solutions are of course possible, and they have been constructed in the literature [3], but they contain sigularities and hence they are not of interest to us.

(iii) Atiyah-Hitchin metric : This metric also has $\lambda_{1}=\lambda_{2}=\lambda_{3}=1$, as in the case of Taub-NUT, but the metric coefficients are not all positive; $a, b$ are positive, while $c$ is taken negative. A particularly useful parametrization of the corresponding line element is $[6,7]$

$$
d s_{A H}^{2}=\frac{1}{4} a^{2} b^{2} c^{2}\left(\frac{d k}{k k^{\prime 2} K^{2}}\right)^{2}+a^{2}(k) \sigma_{1}^{2}+b^{2}(k) \sigma_{2}^{2}+c^{2}(k) \sigma_{3}^{2},
$$

where $a, b, c$ are given as functions of $k$,

$$
\begin{aligned}
a b & =-K(k)(E(k)-K(k)), \\
b c & =-K(k)\left(E(k)-k^{\prime 2} K(k)\right), \\
a c & =-K(k) E(k) .
\end{aligned}
$$

Here, $K(k)$ and $E(k)$ are the complete elliptic integrals of the first and second kind respectively, with $0<k<1$, and ${k^{\prime}}^{2}=1-k^{2}$ is the complementary modulus. The coordinate $t$ in the parametrization (4) is given by the change of variables

$$
t=-\frac{2 K\left(k^{\prime}\right)}{\pi K(k)}
$$

up to an additive constant. The verification of equations (5) follows from the differential equations obeyed by the complete elliptic integrals. We also note using standard expansions of the elliptic integrals that the Atiyah-Hitchin metric approaches the Taub-NUT limit as $k \rightarrow 1$, in which case one obtains the Taub-NUT metric with negative moduli (mass) parameter $m=-1 / 2$. 
Having presented the complete list of the $S O(3)$-invariant hyper-Kahler 4-metrics, we are now in the position to examine their Toda frame formulation that exploits the presence of rotational isometries and leads to the explicit construction of all three complex structures as an $S O(2)$-doublet plus a singlet. Recall first the precise definition that distinguishes a rotational from a translational (also known as tri-holomorphic) Killing vector field $K_{\mu}$. If $K_{\mu}$ satisfies the condition

$$
\nabla_{\nu} K_{\mu}= \pm \frac{1}{2} \sqrt{\operatorname{det} g} \epsilon_{\nu \mu}^{\kappa \lambda} \nabla_{\kappa} K_{\lambda}
$$

it will be called translational, otherwise it will be rotational $[9,10]$. Here, the \pm sign is chosen according to the self-dual or the anti-self-dual nature of the underlying 4-metric $g_{\mu \nu}$. A more algebraic description of the character of a Killing vector field, which will be useful in the following, is given in terms of adapted coordinates for the metric

$$
d s^{2}=V\left(d \tau+\omega_{i} d x^{i}\right)^{2}+V^{-1} \gamma_{i j} d x^{i} d x^{j}
$$

where $V, \omega_{i}, \gamma_{i j}$ are all independent of $\tau$ and $K=\partial / \partial \tau$, using the notion of the nut potential. The nut potential $b_{n u t}$ of a generic vacuum metric (14) that satisfies Einstein's equations is defined using the isometry $\partial / \partial \tau$, as follows:

$$
\partial_{i} b_{n u t}=\frac{1}{2} V^{2} \sqrt{\operatorname{det} \gamma} \epsilon_{i}^{j k}\left(\partial_{j} \omega_{k}-\partial_{k} \omega_{j}\right)
$$

For rotational isometries generated by $\partial / \partial \tau$, the quantity

$$
S_{ \pm}=b_{n u t} \pm V
$$

is coordinate dependent (it will be constant only for translational isometries), and it can always be chosen equal to one of the coordinates, say $x^{3}=z$, up to an overall normalization given by $1 / \sqrt{\gamma^{i j}\left(\partial_{i} S_{ \pm}\right)\left(\partial_{j} S_{ \pm}\right)}$. Then, the other two coordinates $x^{1}=x$ and $x^{2}=y$ can be chosen so that without loss of generality the metric (14) has elements

$$
V^{-1}=\partial_{z} \Psi, \quad \omega_{1}=\mp \partial_{y} \Psi, \quad \omega_{2}= \pm \partial_{x} \Psi, \quad \omega_{3}=0,
$$

and diagonal $\gamma$-metric

$$
\gamma_{11}=\gamma_{22}=e^{\Psi}, \quad \gamma_{33}=1
$$

all of which are determined in terms of a single scalar function $\Psi(x, y, z)[9,10]$. With this choice, which is possible only for hyper-Kahler metrics, the function $\Psi$ satisfies the so-called 3-dim continual Toda equation

$$
\left(\partial_{x}^{2}+\partial_{y}^{2}\right) \Psi+\partial_{z}^{2}\left(e^{\Psi}\right)=0
$$

The continual Toda equation arises as a large $N$ limit of the ordinary 2-dim Toda theory based on the group $S U(N)$, where the Dynkin diagram becomes a continuous line parametrized by the third space variable $z[11,12]$. In the context of general relativity, this equation first appeared in the work of Boyer and Finley [9]. 
Our aim is to examine the rotational versus the translational character of the Killing vector fields of the above three $S O(3)$-invariant hyper-Kahler 4-metrics [8], and use this formalism to construct explicitly the corresponding Toda potentials of the metrics. The common element of these three metrics is the presence of (at least one) rotational isometry, and this is where our analysis will come into play. In particular, one finds that the $S O(3)$ generators of the Eguchi-Hanson metric are translational, while the additional $S O(2)$ isometry $\partial / \partial \psi$ coming from $a^{2}=b^{2}$ is rotational. For the Taub-NUT metric the situation is reversed with the $S O(3)$ generators acting as rotational isometries and $\partial / \partial \psi$ being translational. Finally, for the Atiyah-Hitchin metric the generators of the $S O(3)$ isometry are rotational; in fact this solution provides the only example known to this date of a complete hyper-Kahler 4-metric that is purely rotational without exhibiting any translational isometries. We now list the relevant coordinate transformations that bring these three metrics into their respective Toda frames, and choose our conventions so that the relevant object to consider is $S_{+}$instead of $S_{-}$. Further details can be found in the report of our recent work [13].

(i) Eguchi-Hanson metric : Using the rotational Killing vector field $\partial / \partial \psi$, we write the metric (6) in the form (14) and determine the explicit expression for $V$ and $b_{\text {nut }}$. This allows to find the coordinate $z$ according to the general theory we have outlined. The complete transformation to the Toda frame (17)-(18) can be easily performed thanks to the diagonal form of the metric. The result is summarized as follows:

$$
\begin{aligned}
& x=2 \sqrt{2} \cos \phi \tan \frac{\theta}{2}, \quad y=2 \sqrt{2} \sin \phi \tan \frac{\theta}{2}, \\
& z=\frac{1}{4} r^{2}, \quad \tau=2(\psi+\phi) .
\end{aligned}
$$

Then, the corresponding Toda potential turns out to be

$$
e^{\Psi}=\frac{z^{2}-\alpha^{2}}{2\left(1+\frac{1}{8}\left(x^{2}+y^{2}\right)\right)^{2}}, \quad z^{2} \geq \alpha^{2},
$$

where $4 \alpha=m^{2}$, and this is clearly a solution of the continual Toda equation (19). The presence of the other isometry $\partial / \partial \phi$ reflects into the symmetric quadratic dependence of $\Psi$ on $x$ and $y$.

(ii) Taub-NUT metric : For the Taub-NUT metric we may use $\partial / \partial \phi$ as the generator of a rotational isometry, and after some calculation we find that the change of coordinates that brings the line element (8) into a manifest Toda frame form is given by

$$
\begin{aligned}
& x=\psi, \quad y=-\frac{1}{4 m^{2} t} \cos \theta+\log \left(\tan \frac{\theta}{2}\right), \\
& z=\frac{1}{4 t}\left(1+\frac{1}{8 m^{2} t} \sin ^{2} \theta\right), \quad \tau=2 \phi .
\end{aligned}
$$

Here, the coordinate $t$ is related to the standard variable $r$ by equation (9). The Toda potential of this metric turns out to be

$$
e^{\Psi}=\frac{1}{16 t^{2}} \sin ^{2} \theta
$$


We remark that the presence of the additional $S O(2)$ isometry $\partial / \partial \psi$ reflects into the form of $\Psi$ as $x$-independence. This Toda potential is also a solution of the non-linear equation (19), as it is required on general grounds, but it can not be expressed as an explicit function of $y$ and $z$ in closed form.

(iii) Atiyah-Hitchin metric : Like the Taub-NUT metric, we also use here the rotational character of the Killing vector field $\partial / \partial \phi$ in order to perform the transformation to a Toda frame. The result, which is much more complicated in comparison to the Eguchi-Hanson and Taub-NUT metrics, was first presented in the literature by Olivier [14]. Using our notation, and introducing the complex variable

$$
\nu=\log \left(\tan \frac{\theta}{2}\right)+i \psi,
$$

the relevant transformation to the Toda frame reads:

$$
\begin{gathered}
y+i x=K(k) \sqrt{1+k^{\prime 2} \sinh ^{2} \nu}\left(\cos \theta+\frac{\tanh \nu}{K(k)} \int_{0}^{\pi / 2} d \gamma \frac{\sqrt{1-k^{2} \sin ^{2} \gamma}}{1-k^{2} \tanh ^{2} \nu \sin ^{2} \gamma}\right), \\
z=\frac{1}{8} K^{2}(k)\left(k^{2} \sin ^{2} \theta+k^{\prime 2}\left(1+\sin ^{2} \theta \sin ^{2} \psi\right)-2 \frac{E(k)}{K(k)}\right),
\end{gathered}
$$

and

$$
\tau=2\left(\phi+\arg \left(1+k^{\prime 2} \sinh ^{2} \nu\right)\right)
$$

We also obtain the Toda potential of the Atiyah-Hitchin metric,

$$
e^{\Psi}=\frac{1}{16} K^{2}(k) \sin ^{2} \theta\left|1+k^{\prime 2} \sinh ^{2} \nu\right|
$$

It can be easily verified that in the limit $k \rightarrow 1$ the above expressions yield the corresponding results for the Taub-NUT metric. It is also impossible here to write $\Psi$ as an explicit function of $x, y$ and $z$ in closed form.

We now turn to the problem of the explicit consruction of all three complex structures, using the Toda frame formulation. In a system like the Toda frame, which uses local coordinates that are adapted to a rotational isometry, the corresponding three Kahler forms are not all invariant under $\tau$-shifts. This is another way of saying that rotational isometries have a non-triholomorphic action. In fact, the Kahler forms in this case group into an $S O(2)$-doublet plus a singlet, which can be written down explicitly [2] as follows: The doublet is

$$
\left(\begin{array}{c}
F_{1} \\
F_{2}
\end{array}\right)=e^{\frac{1}{2} \Psi}\left(\begin{array}{cc}
\cos \frac{\tau}{2} & \sin \frac{\tau}{2} \\
\sin \frac{\tau}{2} & -\cos \frac{\tau}{2}
\end{array}\right)\left(\begin{array}{l}
f_{1} \\
f_{2}
\end{array}\right)
$$

where

$$
\begin{aligned}
& f_{1}=\left(d \tau+\omega_{2} d y\right) \wedge d x-V^{-1} d z \wedge d y \\
& f_{2}=\left(d \tau+\omega_{1} d x\right) \wedge d y+V^{-1} d z \wedge d x
\end{aligned}
$$


while the singlet is

$$
F_{3}=\left(d \tau+\omega_{1} d x+\omega_{2} d y\right) \wedge d z+V^{-1} e^{\Psi} d x \wedge d y .
$$

If we were considering the action of the whole $S O(3)$ isometry on the three Kahler forms, it would turn out that either they form a triplet when all the generators are rotational, as in Taub-NUT and Atiyah-Hitchin metrics, or they are all singlets when the generators are translational, as in the Eguchi-Hanson metric [8].

The complex structures of the Eguchi-Hanson and Taub-NUT metrics are already known in the literature, because these metrics exhibit (at least) one translational isometry, and there are simple formulas for them in adapted translational coordinates (see for instance [8]). Nevertheless, we may use the above result to find the form of the three complex structures in the Toda frame formulation of these two metrics as well. However, for the Atiyah-Hitchin metric, which is purely rotational, the use of our result provides a way to perform the explicit construction of all three Kahler forms. It is straightforward, but a very tedious exercise, to use the transformations (25)-(28) and obtain the result for $F_{1}, F_{2}, F_{3}$ directly in terms of the original variables $\theta, \phi, \psi$ and $k$ of the Atiyah-Hitchin metric. There is also an alternative explicit construction of the complex structures as an $S O(3)$-triplet. We hope to make available the details of these expressions in another publication [15].

In conclusion, note that our study of rotational hyper-Kahler geometry has so far been limited to the class of $S O(3)$-invariant metrics. The Atiyah-Hitchin metric is very special, exhibiting only rotational isometries, and it can be regarded as the simplest representative from a class of purely rotational metrics. The construction of a descending series of new 4-dim SO(2)-invariant hyper-Kahler metrics, if they indeed exist, will certainly require a deeper understanding of the special features of the Atiyah-Hitchin metric, using many different points of view. One way to understand the qualitative differences of the Atiyah-Hitchin metric from the other two solutions is provided by the free field realization of the corresponding Toda potentials. An investigation along these lines will be reported elsewhere [13]. We only mention here that a drawback of our method is the local nature of the Toda construction; although every solution of the continual Toda equation corresponds to a 4-dim hyper-Kahler metric, the issue of their completeness requires a separate and more delicate study.

\section{Acknowledgements}

One of us (I.B.) wishes to thank the organizers of the Buckow Symposium for their kind invitation and financial support that made possible his participation to this very enjoyable event. 


\section{REFERENCES}

1. N. Hitchin, A. Karlhede, U. Lindstrom and M. Rocek, Comm. Math. Phys. $\underline{108}$ (1987) 535.

2. I. Bakas and K. Sfetsos, Phys. Lett. B349 (1995) 448.

3. G. Gibbons and C. Pope, Comm. Math. Phys. $\underline{66}$ (1979) 267.

4. T. Eguchi, P. Gilkey and A. Hanson, Phys. Rep. $\underline{66}$ (1980) 213.

5. N. Manton, Phys. Lett. B110 (1982) 54; in "Monopoles in Quantum Field Theory", World Scientific, Singapore, 1981.

6. M. Atiyah and N. Hitchin, Phys. Lett. A107 (1985) 21; Phil. Trans. R. Soc. Lond. A315 (1985) 459; "The Geometry and Dynamics of Magnetic Monopoles", Princeton University Press, Princeton, 1988.

7. G. Gibbons and N. Manton, Nucl. Phys. B274 (1986) 183.

8. G. Gibbons and P. Rubback, Comm. Math. Phys. 115 (1988) 267.

9. C. Boyer and J. Finley, J. Math. Phys. $\underline{23}$ (1982) 1126.

10. J. Gegenberg and A. Das, Gen. Rel. Grav. 16 (1984) 817.

11. M. Saveliev, Comm. Math. Phys. 121 (1989) 283; Theor. Math. Phys. $\underline{92}$ (1993) 1024.

12. I. Bakas, in "Supermembranes and Physics in 2+1 Dimensions", eds. M. Duff, C. Pope and E. Sezgin, World Scientific, Singapore, 1990; Comm. Math. Phys. 134 (1990) 487; Q.-H. Park, Phys. Lett. B238 (1990) 287.

13. I. Bakas and K. Sfetsos, "Toda Fields of $S O(3)$ Hyper-Kahler Metrics and Free Field Realizations", CERN/ENSLAPP/Utrecht preprint (to appear).

14. D. Olivier, Gen. Rel. Grav. 23 (1991) 1349.

15. K. Sfetsos, Utrecht preprint (to appear). 